Int. J. Morphol.,

33(4):1348-1354, 2015

\title{
Biología del Desarrollo Vascular: Mecanismos en Condiciones Físiológicas y Estrés Flujo
}

\author{
Biology of Vascular Development: Mechanisms in Physiological Conditions and Shear Stress
}

\author{
Iván Rodríguez-Núñez ${ }^{*, * * * * *}$; Fernando Romero ${ }^{* * * * * * *} ;$ Marcelo González $^{* * * * *}$ \& Ruy R. Campos ${ }^{* * * * * *}$
}

RODRÍGUEZ-NÚÑEZ, I.; ROMERO, F.; GONZÁLEZ, M. \& CAMPOS, R. R. Biología del desarrollo vascular: mecanismos en condiciones físiológicas y estrés flujo. Int. J. Morphol., 33(4):1348-1354, 2015.

RESUMEN: La vasculogénesis es controlada por una serie de mecanismos que se activan en función del tiempo y del espacio durante el desarrollo embrionario. Múltiples son las vías de señalización implicadas en las etapas del proceso vasculogénico, las que se inician con estímulos angiogénicos desde el mesodermo o desde el endodermo para dar origen a los angioblastos (células progenitoras endoteliales). Proteínas como el factor de crecimiento vascular endotelial (VEGF), factor de crecimiento fibroblastico 2 (FGF2), entre otras, constituyen factores claves en la inducción de este proceso. Posteriormente, los angioblastos deben migrar para dar origen a los vasos primitivos, proceso en el que participan factores atrayentes y repulsivos que orientarán la dirección de su migración. Adicionalmente, los mecanismos de diferenciación arterio-venosa, regulados por la vía de señalización Hedgegog, VEGF y Notch, son determinados antes del inicio de la circulación, lo que sugiere que el destino de la célula endotelial se encuentra genéticamente determinado. Por su parte, los procesos de remodelación y proliferación vascular post natal, son generados a través de la formación de nuevos vasos a partir de vasos pre existentes (angiogénesis). El factor angiogénico que induce los cambios morfológicos y funcionales en las células endoteliales es el VEGFA, las cuales, adquieren la capacidad de direccionar al nuevo vaso en desarrollo. Uno de los principales estímulos físicos que modifica el patrón de crecimiento de los lechos vasculares es el estrés de flujo, el cual, es susceptible de ser modificado por situaciones de estrés como el ejercicio físico. En la presente revisión, se abordan los principales mecanismos implicados en la regulación fisiológica de la vasculogénesis y angiogénesis. Adicionalmente, se discutirán los mecanismos que sustentan la respuesta vascular inducida por estrés de flujo, considerando su rol en el establecimiento de los patrones de crecimiento vascular.

PALABRAS CLAVE: Endotelio; Ejercicio; Neovascularización Fisiológica; Moduladores de la Angiogénesis.

\section{INTRODUCCIÓN}

La alta demanda energética generada en etapas tempranas del desarrollo embrionario requiere de un adecuado y creciente sistema de intercambio de gases, nutrientes y desechos metabólicos. Por este motivo, el sistema cardiovascular es de los primeros sistemas en desarrollarse durante la organogénesis (Adams \& Alitalo, 2007; Karamysheva, 2008; Carmeliet \& Jain, 2011). La morfogénesis vascular (vasculogénesis) es controlada por una serie de mecanismos moleculares que se expresan y activan en función del tiempo y del espacio en que ocurre el desarrollo embrionario (Adams \& Alitalo), dando origen a modificaciones metabólicas en las células progenitoras endoteliales, necesarias para el normal desarrollo de las distintas etapas del proceso de vasculogénesis (Eelen et al., 2013).
Por otra parte, muchos de los eventos que tienen lugar durante el desarrollo vascular embrionario son recapitulados en el adulto mediante la angiogénesis desde vasos preexistentes (Asahara \& Kawamoto, 2004). Por este motivo, el abordaje integral de estos procesos permite un adecuado entendimiento de los mecanismos fisiológicos que dan origen a los cambios y adaptaciones vasculares en situaciones de estrés (Yan et al., 2011).

En la presente revisión, se discuten las principales evidencias que permiten la comprensión de los procesos de vasculogénesis y angiogénesis. El foco se orientará en describir los principales mecanismos de señalización involucrados en la regulación fisiológica de estos procesos

* Escuela de Kinesiología, Facultad de Ciencias de la Salud, Universidad San Sebastián, Concepción. Chile.

** Carrera de Kinesiología, Facultad de Salud, Universidad Santo Tomás, Concepción, Chile.

*** Programa de Doctorado en Ciencias Médicas, Facultad de Medicina, Universidad de la Frontera, Temuco, Chile.

***** Laboratorio de Neurociencia y Biología de péptidos, CEBIOR-CEGIN, BIOREN, Universidad de la Frontera, Temuco, Chile.

****** Laboratorio de Fisiología Vascular, Facultad de Ciencias Biológicas, Universidad de Concepción, Concepción, Chile.

******* Fisiologia Cardiovascular, Escola Paulista de Medicina, Universidade Federal do São Paulo, São Paulo, Brasil. 
complejos. Adicionalmente, se discutirán los mecanismos que sustentan la respuesta vascular inducida por estrés de flujo (EF), considerando su rol trascendente en el establecimiento de los patrones de crecimiento vascular.

\section{VASCULOGÉNESIS}

Desde una perspectiva histórica, la vasculogénesis ha sido descrita como un fenómeno característico de la embriogénesis. No obstante, hoy en día se acepta que tanto la angiogénesis como la vasculogénesis ocurren durante tanto en el desarrollo embrionario como en el desarrollo vascular adulto (Asahara \& Kawamoto). Se ha descrito que las células responsables de la formación de estructuras vasculares primitivas se diferencian desde angioblastos, en embriones, y desde células progenitoras endoteliales, mesoangioblastos y células progenitoras multipotentes, en adultos (Schmith et al., 2007). Las etapas esenciales en la vasculogenesis son: diferenciación de células mesodérmicas en angioblastos, migración de angioblastos, ensamblaje de angioblastos en estructuras vasculares, formación de lumen vascular y organización de la red vascular (Chapell \& Bautch, 2010; Schmith et al.).

Diferenciación: Durante el desarrollo embrionario temprano, la vasculogénesis se inicia con la diferenciación de células precursoras endoteliales del mesodermo en hemangioblastos, estas células son progenitoras de células hematopoieticas y angioblastos, que posteriormente formarán una red vascular que dará origen al plexo vascular pri- mitivo (Karamysheva; Vogeli et al., 2006). Estímulos directos como la proteína morfogénica ósea 4 (BMP4) y el factor de crecimiento vascular endotelial (VEGF), así como también estímulos provenientes del endodermo como las proteínas Indian Hedgehog (Ihh) y el factor de crecimiento de fibroblastos 2 (FGF2), inducen la diferenciación directa de células mesodérmicas en angioblastos (Chapell \& Bautch). Se ha descrito que la señalización sinérgica entre Ihh y FGF2 provenientes del endodermo, promueve la expresión del receptor FLK1 (VEGFR-2) en el mesodermo adyacente. Esto permite que las células progenitoras mesodérmicas respondan a VEGF, lo cual es el punto de partida de la cascada de vasculogénesis (Goldie et al., 2008) (Fig. 1).

Migración: Las células diferenciadas migran desde la línea posterior primitiva al ectodermo extraembrionario, saco vitelino y alantoides, y ectodermo embrionario (Chung \& Ferrara, 2011). Este proceso de migración angioblástica es dependiente del endodermo, el cual permite que los angioblastos desactiven sus uniones célula-célula y migren como una célula única (Jin et al., 2005). Por otra parte, en etapas tardías de la embriogénesis, los angioblastos migran desde fuentes epiteliales o mesenquimales en órganos ya desarrollados, tal como ojos y corazón (Schmith et al.).

Las vías de señalización asociadas a VEGF juegan un rol central en la migración de los angioblastos. Se ha demostrado que el factor de crecimiento vascular endotelial A (VEGF-A) se concentra en la región anterior del embrión, lo cual, es crucial para la movilización, desde la zona poste-

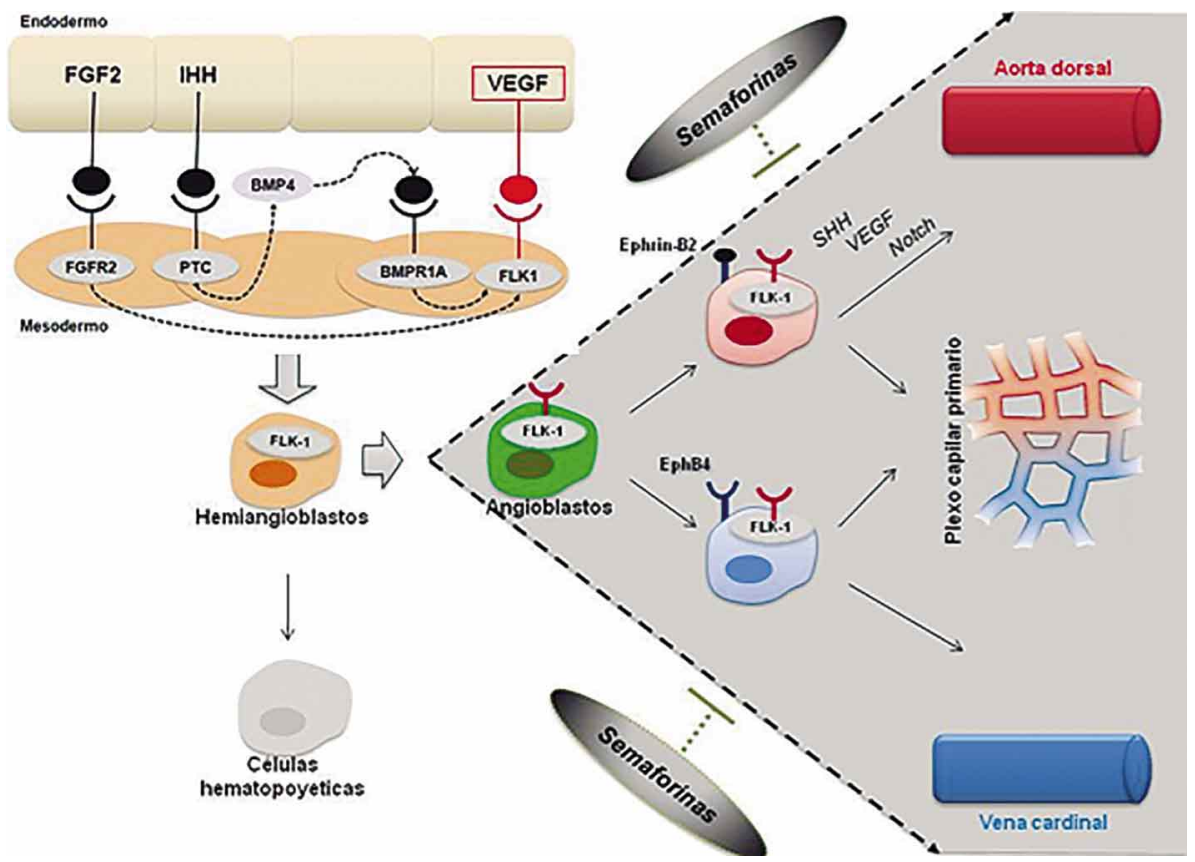

Fig. 1. Mecanismo de vasculogénesis (Ver texto) (Modificado de Chung \& Ferrara, 2011). 
rior, de aquellas células que expresan el VEGFR 1 y 2 (Hiratsuka et al., 2005). Por otra parte, se ha observado en un modelo de ratón knockout, que la nula expresión de VEGFR 1 y 2 genera la muerte del animal en el día 8 de desarrollo embrionario. Adicionalmente, aquellos que presentan nula expresión de VEGFR1 presentan un ensamblaje celular alterado y canal vascular anormal, al poseer interacciones celula-celula y celula-matriz inestables; mientras que aquellos carentes de VEGFR2 fallecen tempranamente, sin diferenciación angioblástica ni desarrollo vascular (Fong et al., 1995; Shalaby et al., 1995).

También existen factores atrayentes y repulsivos asociados a la migración de los angioblastos. Entre estos factores se encuentran las semaforinas, una extensa familia de genes altamente conservados en distintas especies ( $\mathrm{He}$ et al., 2002); entre ellas, la proteína Sema3a fue el primer miembro de esta familia en ser considerado repulsivo para los conos de crecimiento neuronal (Goshima et al., 2000).

El receptor de Sema3a consiste en un complejo de moléculas plexinas y neurofilinas, las cuales se integran con el VEGFR-2 para dar origen al receptor de una isoforma de VEGF, el VEGF165. En este contexo, Shoji et al. (2003) observaron en embriones del pez cebra, que angioblastos positivos para neurofilina-1 migran a regiones donde Sema 3a1 no se encuentra expresada; a su vez, demostraron que el bloqueo en la expresión de Sema3a1, interfiere con la migración de angioblastos y afecta negativamente al desarrollo de la aorta dorsal y circulación sanguínea (Fig. 1).

Diferenciación arterio-venosa: El ensamblaje de las células precursoras en los vasos primarios axiales del tronco, la aorta dorsal (AD) y la vena cardinal posterior (VCP) del pez cebra, sugiere que la expresión de marcadores de identidad venosa o arterial ocurre en etapas tempranas de la vasculogénesis, incluso antes del inicio del latido cardiaco y flujo sanguíneo, lo cual demuestra que el destino de la célula endotelial es genéticamente programado (Swift \& Weinstein, 2009). En este sentido, se ha observado que genes como efirina B2 y EphB4 se expresan en endotelio arterial y venoso respectivamente, antes que la circulación esté totalmente madura (Wang et al., 1998). Los genes EphB4 y efirina B2, dan origen a 2 proteínas integrales de membrana, las cuales requieren del contacto célula-célula para una adecuada señalización celular (Swift \& Weinstein). En este contexto, se ha observado que defectos en la expresión, tanto de efirina como de EphB4, altera significativamente la intercalación entre vasos venosos y arteriales, comprometiendo el establecimiento de los límites entre ellos y la remodelación de la red vascular. No obstante, no pareciera verse afectado el destino celular arterial o venoso; lo cual sugiere la participación de otros factores corriente arriba (Helbling et al., 2000).
Se ha demostrado que las vías de señalización Notch son necesarias para el destino arterial de la célula endotelial. Se ha observado en AD de embriones de pez cebra, que la supresión de esta ruta reduce la expresión del marcador de destino celular arterial efirina B2. Por otra parte, la activación de la vía Notch suprime el destino venoso de las células endoteliales. Resultados que muestran la importancia de la vía de señalización Notch en el proceso de diferenciación (Lawson et al., 2001).

Por su parte, tanto la familia de morfogenes secretados Hedgegog (Hh) como el VEGF, han sido considerados factores corriente arriba de Notch en los procesos de diferenciación vascular (Swift et al.). Lawson et al. (2002) demostraron en pez cebra que sonic hedgehog (Shh) y VEGF actúa corriente arriba de las vías de señalización Notch, durante la diferenciación endotelial arterial. A su vez, evidenciaron que Shh induce la expresión de VEGF, al observar que embriones carentes de Shh no expresan el ARNm de VEGF en DA; el cual, es restaurado posterior a la introducción del ARNm de Shh. Adicionalmente, estudios genéticos en pez cebra han revelado que fosfolipasa C (PLC) g1 es un transductor corriente debajo de la ruta de señalización iniciada por VEGF, el cual es necesario para el proceso de diferenciación arterial (Fig. 1).

En este contexto, dos rutas de señalización intracelular, activadas por el receptor de VEGF, han sido reconocidas: (1) fosfoinositol 3 kinasa (PI3K) y (2) kinasa regulada por señal extracelular / proteína kinasa activada por mitógeno (ERK/ MAPK); las cuales desarrollan roles antagónicos en el proceso de diferenciación vascular (Hong et al., 2008). Se ha observado que la inhibición de la vía de señalización mediada por PI3K promueve la especificación arterial. A la inversa, la inhibición de la vía ERK/MAPK bloquea la especificación arterial y promueve la especificación venosa (Hong et al., 2006). Esto indica que VEGF activa la vía PI3K/Akt, y a su vez, antagoniza la vía PLCg1-ERK/MAPK para favorecer el destino arterial de la célula endotelial (Hong et al., 2008). En adición a lo anterior, se ha observado en respuesta a la inducción de la vía ERK/MAPK, la expresión de un factor de transcripción de destino venoso denominado chicken ovalbumin upstream promoter-transcription factor II (COUP-TFII); el cual, al suprimir su expresión, induce marcadores arteriales en territorio venoso, como ephrinB2, Jag1, Notch1 y NP1 en roedores (Swift et al.).

\section{ANGIOGÉNESIS}

Una vez que el endotelio primitivo ha sido formado, el proceso siguiente es la remodelación, lo cual permite el establecimiento de un red circulatoria madura Este proceso contempla múltiples cascadas de señalización, así como me- 
canismos de comunicaciones célula-célula y célula-matriz extracelular (Goldie et al.; Schmidt et al.). En etapas avanzadas del desarrollo fetal y post natal, una vez que la red vascular primitiva ha sido establecida, la formación de nuevos vasos sanguíneos ocurre vía angiogénesis, definida como la formación de nuevos vasos sanguíneos desde vasos preexistentes. Este proceso es desarrollado a través de 4 diferentes mecanismos: brotes angiogénicos, intususcepción, elongación/ampliación e incorporación de células precursoras circulantes en vasos sanguíneos. De estos mecanismos, el brote angiogénico es uno de los mecanismos mejor descrito (Potente et al., 2011) (Fig. 2A).

Las células endoteliales y células murales comparten una membrana basal conformada por proteínas de ma-
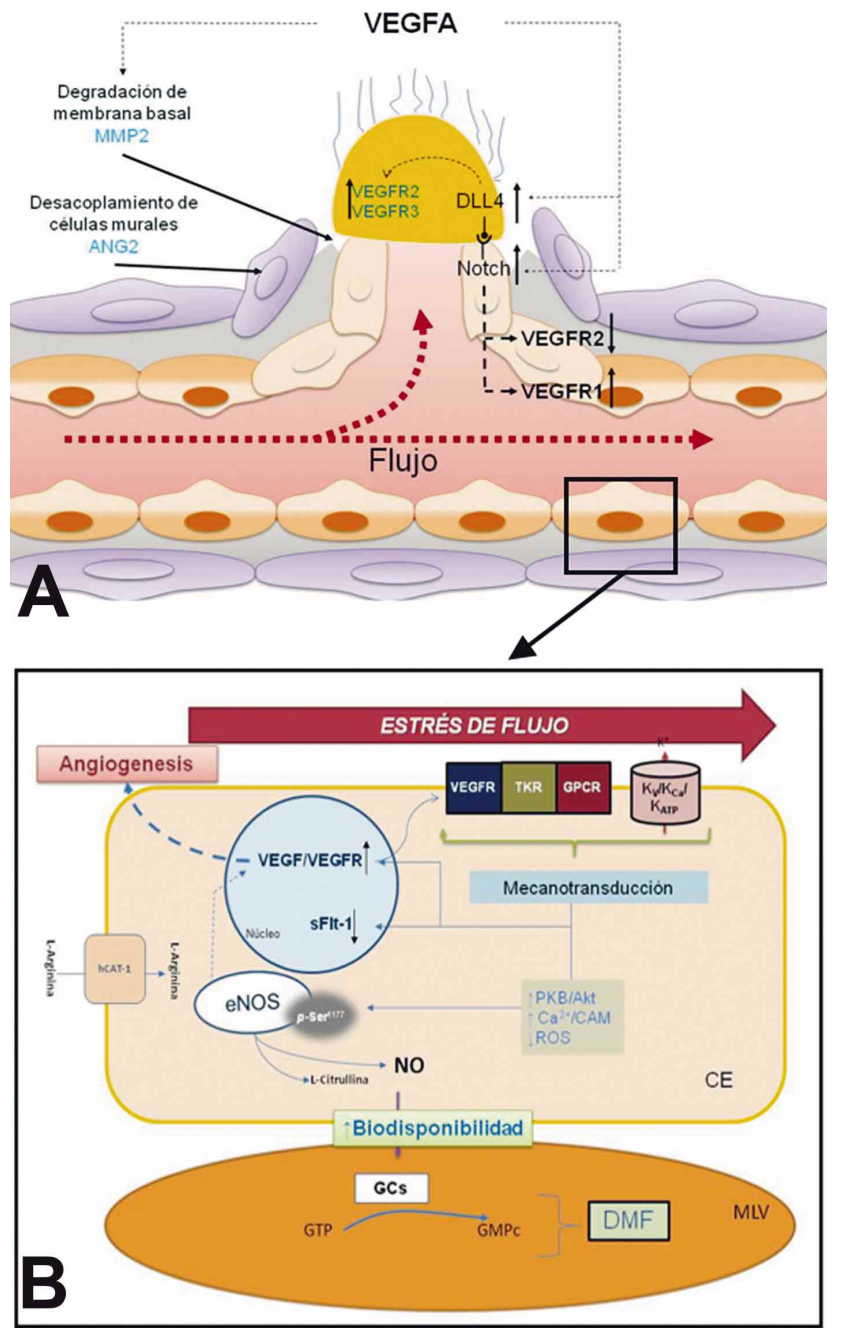

Fig. 2. Angiogénesis. (Modificado de Rodríguez \& González, 2014). En A mecanismo de ramificación arterial (ver texto) y en B respuesta vascular inducida por estrés de flujo (ver texto). $\mathrm{CE}=$ célula endotelial; $\mathrm{MLV}=$ músculo liso vascular; $\mathrm{DMF}=$ dilatación mediada por flujo; $\mathrm{GCs}=$ guanilato ciclasa soluble. triz extracelular que mantiene la conformación de la vasculatura en estado estable. Por este motivo, uno de los primeros eventos que acontecen en respuesta a VEGF, es la estimulación de las metaloproteinasas (MMPs) de matriz extracelular y angiopoyetina-2 (ANG2), que produce la degradación de la membrana basal y estimula el desacoplamiento de las células murales respectivamente (Potente $e t$ al.; Augustin et al., 2009). Posteriormente, se producen cambios morfológicos y funcionales en las células endoteliales, las cuales adquieren movilidad y emiten evaginaciones (filopodias y lamelopodias), dando origen a las células punta (Potente et al., 2011). Estas células generan señales direccionales para la ramificación vascular, y en ellas se produce la interacción de VEGFA con KDR, señal que guiará la ramificación vascular. En respuesta a señales pro angiogénicas, estas células expresan el ligando del receptor Notch, DLL4 (Gerhardt et al., 2003). Por otra parte, las células tallo son inducidas desde las células endoteliales subyacentes a la célula punta, en respuestas a VEGFA. Establecen un lumen y proliferan para favorecer la elongación de la rama vascular; a su vez, expresan el receptor Notch, el cual interactúa con el ligando DLL4 durante la ramificación (Potente et al.). La presencia de VEGF-A incrementa la expresión de DLL4 en las células punta, lo cual incrementa la expresión de los receptores VEGFR-2 y VEGFR-3, dando inicio al proceso angiogénico (Hellström et al., 2007). Por su parte, la activación del receptor Notch en las células tallo disminuye la expresión de VEGFR-2 e incrementa la expresión de VEGFR-1, esto reduce la actividad de VEGF e inhibe la proliferación de las células vecinas (mecanismo de inhibición lateral) (Chappell \& Bautch) (Fig. 2A). Por otra parte, el incremento en la expresión de Jagged1 en las células tallo disminuye la actividad de DLL4 en células punta vecinas; lo que suprime la inducción de la ruta de señalización Notch en células tallo adyacentes, permitiendo direccionar la ruta angiogénica (Benedito et al., 2009) (Fig. 2A).

\section{ESTRÉS DE FLUJO Y ANGIOGÉNESIS}

El estrés de flujo (EF), constituye uno de los principales estímulos físicos sobre la pared vascular, razón por la cual, modifica significativamente el patrón de crecimiento de los lechos vasculares. Hoy en día es aceptado que en condiciones de EF prolongado se produce un incremento de la relación capilar/fibra muscular $(\mathrm{C}: \mathrm{F})$. Contrario a lo que ocurre en condiciones de isquemia donde disminuye la tasa de angiogénesis y el diámetro arteriolar (Hudlicka \& Brown, 2009).

El EF influye significativamente sobre la célula endotelial, por lo cual es necesaria la presencia de mecanismos que permitan transformar el estímulo mecánico en señales químicas intracelulares que inducirán la activación de 
diversas vías de señalización para provocar respuestas adaptativas a nivel genómico y proteómico (Rodríguez \& González, 2014). Existen diversos mecanosensores ubicados en la membrana celular de la célula endotelial, entre ellos destacan los canales iónicos (Gautam et al., 2006), tales como el canal rectificador interno de potasio 2.1 (Kir2.1) (Hoger et al., 2002) y los canales rectificadores externos de Cl- (ORCC) (Lieu et al., 2004). Por otra parte, las caveolas, pequeñas vesículas de membrana abiertas hacia el lumen vascular, han sido descritas como estructuras sensibles al EF (Boyd et al., 2003; Sonveaux et al., 2004). Las proteínas caveolinas (cav) son parte de su estructura y organización, siendo cav 1 la más importante en endotelio vascular. En adición a cav -1, otras proteínas se encuentran presentes en estas vesículas, tales como receptores tirosina quinasa, receptores acoplados a proteína G, VEGFR y canales de Ca2, los que también participan en los mecanismos de mecanotransducción inducida por EF (Sowa, 2012).

La vías de señalización iniciadas a partir de la mecanotransducción convergen en la activación y expresión de la enzima óxido nítrico sintasa (NOS). Esta enzima, en presencia de oxígeno y NADPH, cataliza la síntesis de NO como co-producto de la transformación del aminoácido Larginia a L-citrulina. Existen 3 isoformas: neuronal (NOS 1), inducible (NOS 2) y endotelial (NOS 3), siendo esta última la isoforma de mayor relevancia en la respuesta al estrés hemodinámico (Luiking et al., 2010). Múltiples estudios han mostrado que el EF genera un aumento en la activación y expresión de eNOS, produciendo un incremento en la biodisponibilidad de NO endotelial (Yang et al., 2001; Rodríguez \& González). Adicionalmente, se ha demostrado que la vía de señalización L-arginina/NO es necesaria para el incremento en la expresión de VEGF inducido por ejercicio. Se ha observado que la suplementación alimentaria de L-arginina incrementa la relación capilar:fibra (C:F) en el músculo soleo y subendocardio del ventrículo izquierdo, de ratas Wistar sometidas a 6 semanas de entrenamiento físico. A su vez, la expresión de VEGF y eNOS en el músculo soleo y subendocardio fue mayor en el grupo entrenado y alimentado con un suplemento de L-arginina que en aquellos sin suplementación; resultados que refuerzan la importancia de la ruta L-arginina/NO en la respuesta vascular inducida por estrés hemodinámico (Suzuki, 2006) (Fig. 2B).

\section{CONCLUSIONES}

En los últimos años, ha existido un avance significativo en el estudio de los mecanismos que sustentan el desarrollo vascular. Esto ha permitido un mayor entendimiento de los procesos vasculogénicos y angiogénicos, tanto en con- diciones fisiológicas como patológicas. No obstante, aun existen preguntas abiertas, principalmente en relación a mecanismos que controlan la especificación celular inicial, fisiología y metabolismo de células murales, mecanotransducción endotelial; entre otros. Temas que siguen incentivando el desarrollo de la investigación en esta área, con profundas implicancias clínicas.

RODRÍGUEZ-NÚÑEZ, I.; ROMERO, F.; GONZÁLEZ, M. \& CAMPOS, R. R. Biology of vascular development: mechanisms in physiological conditions and shear stress. Int. J. Morphol., 33(4):1348-1354, 2015.

SUMMARY: Vasculogenesis is controlled by a number of mechanisms that are activated as a function of time and space during embryonic development. Multiple signaling pathways are involved in the stages of vasculogenic process, which start with angiogenic stimuli from the mesoderm or the endoderm to give rise to angioblasts (endothelial progenitor cells). Proteins such as vascular endothelial growth factor (VEGF), fibroblast growth factor 2 (FGF2), among others, are key factors in the induction of this process. Subsequently, the angioblasts must migrate to give birth to primitive vessels, a process that involves attractive and repulsive factors that guide the direction of their migration. Additionally, arterial and venous differentiation regulated hedgegog signaling pathway, VEGF and Notch are determined before the start of circulation, suggesting that the endothelial cell fate is determined genetically. On the other hand, the processes of remodeling and postnatal vascular proliferation are generated through the formation of new vessels from pre-existing vessels (angiogenesis). The angiogenic factor that induces morphological and functional changes in the endothelial cells is the VEGFA, these vessels acquire the ability to address the new developing vessel. One of the main physical stimuli that modify the growth pattern of the vascular beds is the shear stress, which is modified by exercise. In this review, the main mechanisms involved in the physiological regulation of vasculogenesis and angiogenesis are addressed. Additionally, the mechanisms underlying the vascular response induced by shear stress will be discussed, considering its role in establishing patterns of vascular growth.

KEY WORDS: Endothelium; Exercise; Physiologic Neovascularization; Angiogenesis Modulating Agents.

\section{REFERENCIAS BIBLIOGRAFICAS}

Adams, R. H. \& Alitalo, K. Molecular regulation of angiogenesis and lymphangiogenesis. Nat. Rev. Mol. Cell Biol., 8(6):464$78,2007$.

Asahara, T. \& Kawamoto, A. Endothelial progenitor cells for postnatal vasculogenesis. Am. J. Physiol. Cell. Physiol., 287(3):C572-9, 2004. 
Augustin, H. G.; Koh, G. Y.; Thurston, G. \& Alitalo, K. Control of vascular morphogenesis and homeostasis through the angiopoietin-Tie system. Nat. Rev. Mol. Cell Biol.,10(3):16577, 2009.

Benedito, R.; Roca, C.; Sörensen, I.; Adams, S.; Gossler, A.; Fruttiger, M. \& Adams, R. H. The notch ligands Dll4 and Jagged1 have opposing effects on angiogenesis. Cell, 137(6):1124-35, 2009.

Boyd, N. L.; Park, H.; Yi, H.; Boo, Y. C.; Sorescu, G. P.; Sykes, M. \& Jo, H. Chronic shear induces caveolae formation and alters ERK and Akt responses in endothelial cells. Am. J. Physiol. Heart Circ. Physiol., 285(3):H1113-22, 2003.

Carmeliet, P. \& Jain, R. K. Molecular mechanisms and clinical applications of angiogenesis. Nature, 473(7347):298-307, 2011.

Chappell, J. C. \& Bautch, V. L. Vascular development: genetic mechanisms and links to vascular disease. Curr. Top. Dev. Biol., 90:43-72, 2010.

Chung, A. S. \& Ferrara, N. Developmental and pathological angiogenesis. Annu. Rev. Cell Dev. Biol., 27:563-84, 2011.

Eelen, G.; Cruys, B.; Welti, J.; De Bock, K. \& Carmeliet, P. Control of vessel sprouting by genetic and metabolic determinants. Trends Endocrinol. Metab., 24(12):589-96, 2013.

Fong, G. H.; Rossant, J.; Gertsenstein, M. \& Breitman, M. L. Role of the Flt-1 receptor tyrosine kinase in regulating the assembly of vascular endothelium. Nature, 376(6535):6670,1995

Gautam, M.; Shen, Y.; Thirkill, T. L.; Douglas, G. C. \& Barakat, A. I. Flow-activated chloride channels in vascular endothelium. Shear stress sensitivity, desensitization dynamics, and physiological implications. J. Biol. Chem., 281(48):36492-500, 2006.

Gerhardt, H.; Golding, M.; Fruttiger, M.; Ruhrberg, C.; Lundkvist, A.; Abramsson, A.; Jeltsch, M.; Mitchell, C.; Alitalo, K.; Shima, D. \& Betsholtz, C. VEGF guides angiogenic sprouting utilizing endothelial tip cell filopodia. J. Cell Biol., 161(6):1163-77, 2003

Goldie, L. C.; Nix, M. K. \& Hirschi, K. K. Embryonic vasculogenesis and hematopoietic specification. Organogenesis, 4(4):257-63, 2008.

Goshima, Y.; Sasaki, Y.; Nakayama, T.; Ito, T. \& Kimura, T. Functions of semaphorins in axon guidance and neuronal regeneration. Jpn. J. Pharmacol., 82(4):273-9, 2000.

He, Z.; Wang, K. C.; Koprivica, V.; Ming, G. \& Song, H. J. Knowing how to navigate: mechanisms of semaphorin signaling in the nervous system. Sci. STKE, 2002(119):re1, 2002.
Helbling, P. M.; Saulnier, D. M. \& Brändli, A. W. The receptor tyrosine kinase EphB4 and ephrin-B ligands restrict angiogenic growth of embryonic veins in Xenopus laevis. Development, 127(2):269-78, 2000.

Hellström, M.; Phng, L. K.; Hofmann, J. J.; Wallgard, E.; Coultas, L.; Lindblom, P.; Alva, J.; Nilsson, A. K.; Karlsson, L.; Gaiano, N.; Yoon, K.; Rossant, J.; Iruela-Arispe, M. L.; Kalén, M.; Gerhardt, H. \& Betsholtz, C. Dll4 signalling through Notch1 regulates formation of tip cells during angiogenesis. Nature, 445(7129):776-80, 2007.

Hiratsuka, S.; Kataoka, Y.; Nakao, K.; Nakamura, K.; Morikawa, S.; Tanaka, S.; Katsuki, M.; Maru, Y. \& Shibuya, M. Vascular endothelial growth factor A (VEGF-A) is involved in guidance of VEGF receptor-positive cells to the anterior portion of early embryos. Mol. Cell Biol., 25(1):355-63, 2005.

Hoger, J. H.; Ilyin, V. I.; Forsyth, S. \& Hoger, A. Shear stress regulates the endothelial Kir2.1 ion channel. Proc. Natl. Acad. Sci. USA, 99(11):7780-5, 2002.

Hong, C. C.; Kume, T. \& Peterson, R. T. Role of crosstalk between phosphatidylinositol 3-kinase and extracellular signalregulated kinase/mitogen-activated protein kinase pathways in artery-vein specification. Circ. Res., 103(6):573-9, 2008.

Hong, C. C.; Peterson, Q. P.; Hong, J. Y. \& Peterson, R. T. Artery/ vein specification is governed by opposing phosphatidylinositol-3 kinase and MAP kinase/ERK signaling. Curr. Biol., 16(13):1366-72, 2006.

Hudlicka, O. \& Brown, M. D. Adaptation of skeletal muscle microvasculature to increased or decreased blood flow: role of shear stress, nitric oxide and vascular endothelial growth factor. J. Vasc. Res., 46(5):504-12, 2009.

Jin, S. W.; Beis, D.; Mitchell, T.; Chen, J. N. \& Stainier, D. Y. Cellular and molecular analyses of vascular tube and lumen formation in zebrafish. Development, 132(23):5199-209, 2005.

Karamysheva, A. F. Mechanisms of angiogenesis. Biochemistry (Mosc.), 73(7):751-62, 2008.

Lawson, N.D.; Scheer, N.; Pham, V. N.; Kim, C. H.; Chitnis, A. B.; Campos-Ortega, J. A. \& Weinstein, B. M. Notch signaling is required for arterial-venous differentiation during embryonic vascular development. Development, 128(19):3675-83, 2001.

Lawson, N. D.; Vogel, A. M. \& Weinstein, B. M. sonic hedgehog and vascular endothelial growth factor act upstream of the Notch pathway during arterial endothelial differentiation. Dev. Cell., 3(1):127-36, 2002.

Lieu, D. K.; Pappone, P. A. \& Barakat, A. I. Differential membrane potential and ion current responses to different types of shear stress in vascular endothelial cells. Am. J. Physiol. Cell Physiol., 286(6):C1367-75, 2004. 
Luiking, Y. C.; Engelen, M. P. \& Deutz, N. E. Regulation of nitric oxide production in health and disease. Curr. Opin. Clin. Nutr. Metab. Care, 13(1):97-104, 2010.

Potente, M.; Gerhardt, H. \& Carmeliet, P. Basic and therapeutic aspects of angiogenesis. Cell, 146(6):873-87, 2011.

Rodríguez, I. \& González, M. Physiological mechanisms of vascular response induced by shear stress and effect of exercise in systemic and placental circulation. Front. Pharmacol., 5:209, 2014.

Schmidt, A.; Brixius, K. \& Bloch, W. Endothelial precursor cell migration during vasculogenesis. Circ. Res., 101(2):125-36, 2007.

Shalaby, F.; Rossant, J.; Yamaguchi, T. P.; Gertsenstein, M.; Wu, X. F.; Breitman, M. L. \& Schuh, A. C. Failure of blood-island formation and vasculogenesis in Flk-1-deficient mice. Nature, 376(6535):62-6, 1995.

Shoji, W.; Isogai, S.; Sato-Maeda, M.; Obinata, M. \& Kuwada, J. Y. Semaphorin3a1 regulates angioblast migration and vascular development in zebrafish embryos. Development, 130(14):3227-36, 2003.

Sonveaux, P.; Martinive, P.; DeWever, J.; Batova, Z.; Daneau, G.; Pelat, M.; Ghisdal, P.; Grégoire, V.; Dessy, C.; Balligand, J. L. \& Feron, O. Caveolin-1 expression is critical for vascular endothelial growth factor-induced ischemic hindlimb collateralization and nitric oxide-mediated angiogenesis. Circ. Res., 95(2):154-61, 2004.

Sowa, G. Caveolae, caveolins, cavins, and endothelial cell function: new insights. Front Physiol., 2:120, 2012.

Suzuki, J. L-arginine supplementation causes additional effects on exercise-induced angiogenesis and VEGF expression in the heart and hind-leg muscles of middle-aged rats. J. Physiol. Sci., 56(1):39-44, 2006.

Swift, M. R. \& Weinstein, B. M. Arterial-venous specification during development. Circ. Res., 104(5):576-88, 2009.

Vogeli, K. M.; Jin, S. W.; Martin, G. R. \& Stainier, D. Y. A common progenitor for haematopoietic and endothelial lineages in the zebrafish gastrula. Nature, 443(7109):337-9, 2006.

Wang, H. U.; Chen, Z. F. \& Anderson, D. J. Molecular distinction and angiogenic interaction between embryonic arteries and veins revealed by ephrin-B2 and its receptor Eph-B4. Cell, 93(5):741-53, 1998

Yan, Z.; Okutsu, M.; Akhtar, Y. N. \& Lira, V. A. Regulation of exercise-induced fiber type transformation, mitochondrial biogenesis, and angiogenesis in skeletal muscle. J. Appl. Physiol. (1985), 110(1):264-74, 2011.
Yang, H. T.; Yan, Z.; Abraham, J. A. \& Terjung, R. L. VEGF(121)and bFGF-induced increase in collateral blood flow requires normal nitric oxide production. Am. J. Physiol. Heart Circ. Physiol., 280(3):H1097-104, 2001.

Dirección para Correspondencia:

Iván Rodríguez Núñez

Universidad San Sebastián

Facultad de Ciencias de la Salud

Lientur nº 1457

Concepción

CHILE

Teléfono: 56-41-2487293

Email: ivan.rodriguez@uss.cl

Recibido: 11-01-2015

Aceptado: 08-10-2015 